\title{
Reconhecimento de fala em indivíduos com e sem queixa clínica de dificuldade para entender a fala no ruído
}

\section{Speech recognition in individuals having a clinical complaint about understanding speech during noise or not}

\author{
Karine Thaís Becker ${ }^{1}$, Maristela Julio Costa², Larissa Lautenschlager ${ }^{3}$, Tania Maria Tochetto ${ }^{4}$, Sinéia Neujahr dos Santos 5. \\ 1) Graduação. Fonoaudióloga. \\ 2) Doutora. Fonoaudióloga; Professora Adjunta do Departamento de Fonoaudiologia da Universidade Federal de Santa Maria - UFSM. \\ 3) Mestre. Fonoaudióloga do Centro Auditivo Phonak. \\ 4) Doutora. Fonoaudióloga, Professora Associada do Departamento de Fonoaudiologia da UFSM \\ 5) Mestre. Fonoaudióloga do Centro Auditivo Sonora. \\ Instituição: Universidade Federal de Santa Maria - UFSM \\ Santa Maria / RS - Brasil. \\ Endereço para correspondência: Karine Thaís Becker - Rua Pedro Santini, 177 - apto. 109/C - Nossa Senhora de Lourdes - Santa Maria / RS - Brasil - CEP: $97060-480$ \\ - Telefone: (+55 55) 8406-3292 - E-mail: katthais@ hotmail.com \\ Artigo recebido em 4 de Outubro de 2010. Artigo aprovado em 27 de Março de 2011
}

\section{RESUMO}

Introdução: Estudo clínico e experimental. Indivíduos com audição normal podem ser prejudicados em situações de comunicação desfavoráveis, o que interfere negativamente na inteligibilidade de fala.

Objetivo: Verificar e comparar o desempenho de adultos jovens, normo-ouvintes, com e sem queixa clínica de dificuldade para entender a fala no rú́do, utilizando sentenças como estímulo.

Método: Foram avaliados 50 indivíduos, 21 do sexo masculino e 29 do feminino, com idades entre 19 e 32 anos, normoouvintes, divididos em dois grupos: sem e com queixa clínica de dificuldade de entender a fala no ruído. Utilizando o teste Listas de Sentenças em Português, realizou-se a pesquisa dos Limiares de Reconhecimento de Sentenças no Ruído, com os quais foram obtidas as relações sinal-ruído (S/R). O ruído competitivo foi apresentado a $65 \mathrm{~dB}$ NA.

Resultados: Os valores médios obtidos para as relações $S / R$ na orelha direita, para o grupo sem queixa e o grupo com queixa, foram respectivamente $-6,26 \mathrm{~dB}$ e $-3,62 \mathrm{~dB}$. Para a orelha esquerda, foram $-7,12 \mathrm{~dB}$ e $-4,12 \mathrm{~dB}$. Foi verificada diferença estatisticamente significante tanto na orelha direita quanto na esquerda entre os dois grupos.

Conclusão: Indivíduos normo-ouvintes com queixa clínica de dificuldade de entender a fala em ambientes ruidosos possuem maior dificuldade na tarefa de reconhecimento de sentenças no ruído quando comparados a sujeitos que não relatam essa dificuldade. Assim deve-se incluir na avaliação audiológica de rotina testes que empregam sentenças na presença de ruído competitivo, avaliando de forma mais confiável e eficiente o desempenho do reconhecimento de fala. ACTRN12610000822088

Palavras-chave: audição, percepção da fala, testes de discriminação da fala, ruído.

\section{SUMMARY}

Introduction: Clinical and experimental study. Individuals with a normal hearing can be jeopardized in adverse communication situations, what negatively interferes with speech clearness. Objective: check and compare the performance of normal hearing young adults who have a difficulty in understanding speech during noise or not, by making use of sentences as stimuli.

Method: 50 normal hearing individuals, 21 of whom were male and 29 were female, aged between 19 and 32, were evaluated and divided into two groups: with and without a clinical complaint about understanding speech during noise. By using Portuguese Sentence Lists test, the Recognition Threshold of Sentences during Noise research was performed, through which the signal-to-noise (SN) ratios were obtained. The contrasting noise was introduced at $65 \mathrm{~dB}$ NA.

Results: the average values achieved for SN ratios in the left ear, for the group without a complaint and the group with a complaint, were respectively $6.26 \mathrm{~dB}$ and $3.62 \mathrm{~dB}$. For the left ear, the values were $-7.12 \mathrm{~dB}$ and $-4.12 \mathrm{~dB}$. A statistically significant difference was noticed in both right and left ears of the two groups.

Conclusion: normal hearing individuals showing a clinical complaint about understanding speech at noisy places have more difficulty in the task to recognize sentences during noise, in comparison with the people who do not face such a difficulty. Accordingly, the customary audiologic evaluation must include tests using sentences during a contrasting noise, with a view to evaluating the speech recognition performance more reliably and efficiently. ACTRN12610000822088

Keywords: hearing, speech recognition, speech discrimination tests, noise. 


\section{INTRODUÇÃO}

A habilidade para compreender a fala é um aspecto importante a ser considerado na avaliação audiológica, pois permite analisar a função comunicativa-perceptiva, fornecendo dados sobre como o sujeito entende a mensagem falada em situações de escuta diária (1). Indivíduos com audição normal, em geral, apresentam bom desempenho na maioria destas situações, entretanto, em ambientes ruidosos, podem referir dificuldade para compreender a fala. Isso porque quando a avaliação ocorre no ruído são exigidos vários canais auditivos para atingir o reconhecimento da fala, indicando que informações sensoriais mais detalhadas são necessárias em condições de escuta difícil (2).

Para que a inteligibilidade da fala ocorra com sucesso, é necessário que o reconhecimento das características da mensagem e das características acústicas do ambiente aconteça simultaneamente e de forma integrada (3).

O ruído ambiental está cada vez mais constante no cotidiano das pessoas, muitas vezes não a ponto de ser prejudicial e induzir algum dano à audição, mas de interferir diretamente na compreensão das palavra (4). Analisar a relação entre os níveis audiométricos e a capacidade de reconhecer os sinais de fala de cada indivíduo em particular torna-se, então, fundamental no processo de avaliação audiológica. As queixas frequentes de dificuldades para reconhecer a fala, principalmente em ambientes ruidosos, mesmo em indivíduos considerados audiologicamente normais do ponto de vista quantitativo $(4,5)$, nos leva a pensar de que forma o indivíduo deve ser avaliado para quantificar essa dificuldade.

Em uma avaliação audiológica, as dificuldades na compreensão da fala só podem ser realmente evidenciadas com estímulos de fala que representem uma situação comunicativa (6). Por causa do desafio que esta tarefa representa, sua avaliação fornece informações importantes sobre a capacidade do indivíduo em lidar com a escuta em ambientes ruidosos (1).

Para esse fim, foi elaborado o teste Listas de Sentenças em Português - LSP (7), que utiliza frases como estímulo, as quais podem ser aplicadas tanto em situações de silêncio quanto na presença de ruído competitivo. As sentenças representam melhor as características de uma situação de conversação do que as palavras isoladas e, juntamente com o ruído, permitem a avaliação do reconhecimento de fala, simulando em ambiente clínico, situações semelhantes às do dia-a-dia do indivíduo (1).

O LSP proporciona precisão e objetividade para mensurar habilidades de reconhecimento de fala de um ouvinte como reflexo de seu desempenho em situações auditivas realistas e seus achados são de extrema importância para o diagnóstico clínico mais preciso (6).

Com base nessas considerações, o objetivo do presente estudo foi verificar e comparar o desempenho de adultos jovens, normo-ouvintes, com e sem queixa clínica de dificuldade para entender a fala no ruído, utilizando sentenças como estímulo na presença de um ruído competitivo.

\section{MÉTODO}

Este estudo foi realizado no Laboratório de Próteses Auditivas do Serviço de Atendimento Fonoaudiológico (SAF) da Universidade Federal de Santa Maria (UFSM), no ano letivo de 2009, a partir do Projeto "Pesquisa e base de dados em saúde auditiva", registrado no GAP do Centro de Ciências da Saúde sob o no ${ }^{\circ}$ 19731e aprovado pelo Comitê de Ética em Pesquisa com certificado de $\mathrm{n}^{\circ}$ 0138.0.243.000.06

Foram utilizados como critérios de inclusão: audição dentro dos padrões de normalidade, isto é, limiares de audibilidade inferiores a $25 \mathrm{~dB}$ NA nas frequências de 250 a $8000 \mathrm{~Hz}$ (8) e ausência de comprometimento de orelha média, bem como queixa de zumbido e hiperacusia.

Assim, a amostra ficou constituída de 50 indivíduos adultos jovens, com idades entre 19 e 32 anos, normoouvintes, que referiram ou não dificuldade de compreender a fala no ruído, os quais foram divididos em dois grupos: grupo A, sem queixa para compreender a fala, formado por 26 indivíduos, 14 do sexo masculino e 12 do sexo feminino; e grupo B, com queixa para compreender a fala, formado por 24 indivíduos, 7 do sexo masculino e 17 do sexo feminino.

Todos os participantes eram alunos de graduação ou pós-graduação, socialmente ativos e produtivos.

Após receberem orientações sobre os objetivos, justificativa e metodologia do estudo proposto, os sujeitos assinaram o Termo de Consentimento Livre e Esclarecido. Em seguida foram submetidos à anamnese por meio de um questionário, que colheu informações referentes a dados pessoais, queixas auditivas e história otológica.

A avaliação audiológica foi realizada após a inspeção visual do meato acústico externo e consistiu de: audiometria tonal liminar por via aérea nas frequências de 250 a $8.000 \mathrm{~Hz}$ e por via óssea nas frequências de 500 a $4.000 \mathrm{~Hz}$; pesquisa do limiar de reconhecimento de fala (LRF) e pesquisa do índice percentual de reconhecimento de fala (IPRF). Para a obtenção destas medidas, foram 
utilizados: um audiômetro digital de dois canais, marca Fonix, modelo FA-12, tipo I e fones auriculares tipo TDH39P, marca Telephonics. Ainda foram avaliadas as medidas de imitância acústica (MIA) através da timpanometria e pesquisa dos reflexos acústicos, usando um analisador de orelha média INTERACOUSTIC AZ7, com fone TDH-39 e coxim MX-41, com tom sonda de $220 \mathrm{~Hz}$ a $70 \mathrm{~dB}$ NA, e calibração segundo a norma ISO 389-1991. Os reflexos acústicos foram pesquisados nas frequências de 500, 1000 e $2000 \mathrm{~Hz}$.

Posteriormente foi realizada a pesquisa do limiar de reconhecimento de sentenças no ruído (LRSR) e calculada a relação sinal/ruído (S/R), através da aplicação do teste LSP (7). Esse material apresenta-se gravado em CD e contém oito listas de sentenças e um ruído com espectro de fala, gravados em canais independentes, permitindo a apresentação das sentenças no ruído, com intensidades de apresentação diferentes. As sentenças e o ruído foram apresentados utilizando-se um Compact Disc Player Digital Toshiba - 4149 acoplado ao audiômetro acima descrito.

Antes de iniciar o teste com cada indivíduo, a saída de cada canal do $C D$ foi calibrada através do VU-meterdo audiômetro. O tom de $1 \mathrm{kHz}$ presente no mesmo canal do $C D$ em que estão gravadas as sentenças, bem como o ruído mascarante presente no outro canal, foram colocados no nível zero.
As listas de sentenças e o ruído competitivo foram apresentados de forma monoaural e ipsilateralmente, através de fones auriculares, permitindo a avaliação das orelhas separadamente. As listas de sentenças utilizadas estão descritas na Figura 1.

As sentenças foram aplicadas na seguinte ordem:

a) Apresentação das sentenças de 1 a 10 da lista $1 \mathrm{~A}$, com a presença de ruído competitivo ipsilateralmente, na orelha direita, para familiarização do indivíduo com o teste.

b) Apresentação das sentenças de 11 a 20 da lista 1A, com a presença de ruído competitivo ipsilateralmente, na orelha esquerda, para familiarização do indivíduo com o teste.

c) Apresentação da lista 3B com a presença de ruído competitivo ipsilateralmente, na orelha direita.

d) Apresentação da lista 4B com a presença de ruído competitivo ipsilateralmente, na orelha esquerda.

A intensidade inicial de apresentação da primeira sentença de cada lista foi baseada nos resultados encontrados no treinamento acima descrito, sendo que a intensidade do ruído foi mantida constante a 65 dB NA (9). Assim, a relação $S / R$ inicial foi modificando-se a partir da mudança na intensidade de cada sentença.

Por meio do treinamento, foi possível estabelecer para cada indivíduo o nível de intensidade necessária para

Figura I,

\begin{tabular}{ll}
\hline LISTA IA & LISTA 3B \\
1. Não posso perder o ônibus. & I. Ela acabou de bater o carro. \\
2. Vamos tomar um cafezinho. & 2. É perigoso andar nessa rua. \\
3. Preciso ir ao médico. & 3. Não posso dizer nada. \\
4. A porta da frente está aberta. & 4. A chuva foi muito forte. \\
5. A comida tinha muito sal. & 5. Os preços subiram na segunda. \\
6. Chegueiatrasado para a reunião. & 6. Esqueci de levara bolsa. \\
7. Vamos conversarlá na sala. & 7. Os pães estavam quentes. \\
8. Depois liga pra mim. & 8. Elas jáalugaram uma casa na praia. \\
9. Esquecide pagara conta. & 9. Meu irmão viajou de manhã. \\
10. Os preços subiramontem. & 10. Não encontrei meufilho. \\
11. Ojantar está na mesa. & \\
12. Ascrianças estão brincando. & LISTA 4B \\
13. Choveumuito neste fim-de-semana. & I. Sua mãe pôs o carro na garagem. \\
14. Estou morrendo de saudade. & 2. Oaluno quer assistir ao filme. \\
15. Olhe bemao atravessara rua. & 3. Ainda não pensei no que fazer. \\
16. Preciso pensar comcalma. & 4. Essa estrada é perigosa. \\
17. Guardeio livro na primeiragaveta. & 5. Não pagueia conta do bar. \\
18. Hojeé meu dia de sorte. & 6. Meufilho está ouvindo música. \\
19. Osolestá muito quente. & 7. Achuva inundoua rua. \\
20. Sua mãe acabou de sair de carro. & 8. Amanhã não posso almoçar. \\
& 9. Ela viaja em dezembro. \\
& I0.Vocêteve muita sorte.
\end{tabular}


Tabela I . Média, desvio padrão, valores mínimos, máximos do grupo A, para ambas as orelhas.

\begin{tabular}{lllll}
\hline GrupoA & Média & DP & Mín. & Máx. \\
\hline OD & $-6,26$ & 2,32 & $-3,07$ & $-12,8$ \\
OE & $-7,12$ & 2,42 & $-3,66$ & $-11,77$ \\
\hline
\end{tabular}

Tabela 3.. Médias e resultado do teste Mann Whitney, entre os grupos A e B, para a orelha direita.

\begin{tabular}{llll}
\hline Grupo & $N$ & Média & Valorde $p$ \\
\hline A & 24 & $-6,26$ & \\
B & 26 & $-3,62$ & $0,000037^{*}$ \\
\hline
\end{tabular}

(*) Diferença estatisticamente significante $(p<0,05)$

que este tivesse êxito na primeira sentença de cada lista do teste.

A estratégia utilizada para pesquisar o LRSR foi a sequencial ou adaptativa, ou ainda ascendente-descendente (10). Esta permite mensurar o nível necessário para o indivíduo identificar, de forma correta, aproximadamente 50\% dos estímulos de fala apresentados em uma determinada relação $\mathrm{S} / \mathrm{R}$.

São sugeridos intervalos de $4 \mathrm{~dB}$ até a primeira mudança no tipo de resposta e, posteriormente, intervalos de a presentação dos estímulos de 2 dB entre si até o final da lista (10). Porém, devido às possibilidades técnicas do equipamento disponível para a realização desta pesquisa, foram utilizados intervalos de apresentação das sentenças de $5 \mathrm{~dB}$ e $2,5 \mathrm{~dB}$, respectivamente.

Seguindo essa estratégia, quando o indivíduo foi capaz de reconhecer corretamente o estímulo de fala apresentado, a intensidade do mesmo foi diminuída; caso contrário, sua intensidade foi aumentada. Uma resposta só foi considerada correta quando o indivíduo repetiu, sem nenhum erro ou omissão, toda a sentença apresentada.

É importante mencionar aqui que foi observado, no primeiro estudo realizado com fones auriculares (11), uma diferença de 7 dB entre o volume de gravação dos dois sinais a presentados (fala e ruído), sendo que as sentenças estão gravadas em uma intensidade média de $7 \mathrm{~dB}$ abaixo da intensidade do ruído. Por esta razão, a autora do teste referiu que, nas avaliações realizadas com fones auriculares, é necessário que seja subtraído $7 \mathrm{~dB}$ dos valores de fala observados no dial do equipamento, procedimento este adotado nesta pesquisa.

Os níveis de apresentação das sentenças foram anotados para posterior cálculo da média a partir dos
Tabela 2. Média, desvio padrão, valores mínimos, máximos do grupo $B$, para ambas as orelhas.

\begin{tabular}{lcccc}
\hline Grupo B & Média & DP & Mín. & Máx. \\
\hline OD & $-3,621,72$ & $-1,04$ & $-8,07$ & \\
OE & $-4,12$ & 2,29 & $-0,75$ & $-7,25$
\end{tabular}

Tabela 4. Médias e resultado do teste t Pareado, entre os grupos A e B, para a orelha esquerda.

\begin{tabular}{llll}
\hline Grupo & $N$ & Média & Valorde $p$ \\
\hline A & 24 & $-7,12$ & \\
B & 26 & $-4,12$ & $0,000044 *$ \\
\hline
\end{tabular}

(*)Diferença estatisticamente significante $(p<0,05)$

valores onde houve mudança no tipo de resposta e então, subtraídos os $7 \mathrm{~dB}$, resultando no LRSR. Para a obtenção do valor da relação sinal/ruído (S/R), foi subtraído o nível de intensidade do ruído ( $65 \mathrm{~dB}$ NA) do valor do LRSR. A variável considerada no estudo foi o LRSR, expresso através da relação S/R.

Realizou-se a análise descritiva dos valores e, em seguida, os dados coletados foram submetidos a tratamento estatístico, primeiramente, por meio da análise do comportamento das variáveis. Por ser constatada distribuição não-normal dos dados na orelha direita, foi aplicado o teste Manı Whitney; após constatar distribuição normal dos dados encontrados na orelha esquerda, foi aplicado o teste $t$ Pareado. Ambos os testes tem como finalidade comparar se a diferença entre as médias das relações S/R entre os grupos com e sem queixa tiveram significância ou não. Foi considerado nível de significância estatística de $\mathrm{p} \leq 0,05(5 \%)$.

\section{Resultados}

A seguir, estão apresentados os resultados obtidos com as avaliações realizadas nos 50 indivíduos, sendo 24 sem queixa clínica para compreender a fala no ruído (Grupo A) e 26 com queixa (Grupo B).

Na análise estatística, não foi evidenciada diferença estatisticamente significante quanto ao sexo, portanto esta variável foi desconsiderada.

Nas Tabelas 1 e 2 estão expostos os resultados da relação $\mathrm{S} / \mathrm{R}$ de cada grupo.

Nas Tabelas 3 e 4 estão expostos os dados obtidos através da análise comparativa da relação S/R média por orelha, encontrada para cada grupo. 


\section{DISCUSSÃO}

Os valores médios obtidos para as relações S/R na orelha direita, para o grupo A (sem queixa) e grupo B (com queixa), foram de $-6,26 \mathrm{~dB}$ e $-3,62 \mathrm{~dB}$, respectivamente. Já para a orelha esquerda foram $-7,12 \mathrm{~dB}$ e $-4,12 \mathrm{~dB}$.

Com base nesses resultados, pode-se verificar que os valores médios das relações S/R obtidos para os indivíduos que não apresentam queixa foram melhores que os valores médios das relações S/R obtidos para os indivíduos que apresentam queixa de entender a fala no ruído. Isso evidencia que os sujeitos do grupo A conseguiram reconhecer em torno de $50 \%$ dos estímulos de fala apresentados diante de ruído competitivo (65 dB NA) com uma relação S/R mais desfavorável, ou seja, o estímulo de fala foi apresentado em intensidades menores em relação ao ruído.

Segundo a literatura, indivíduos com audição normal podem ser prejudicados em situações de comunicação nas quais a relação $\mathrm{S} / \mathrm{R}$ for desfavorável e interferir negativamente na inteligibilidade de fala (2).

Tal fato foi verificado no presente estudo, pois, ao comparar os resultados entre os grupos, foi verificada diferença estatisticamente significante, tanto na orelha direita quanto na esquerda, revelando que o grupo com queixa (B) teve desempenho significantemente pior se comparado ao grupo sem queixas (A).

Resultados semelhantes foram encontrados por outros pesquisadores $(12,13,14)$.

O grupo A teve, em média, uma relação S/R -2,64 $\mathrm{dB}$ melhor que o grupo $\mathrm{B}$ na OD e $-3 \mathrm{~dB}$ melhor na OE. Testes de sentenças com ruído competitivo podem evidenciar pequenas mudanças na relação $S / R$, traduzindo-as em grandes mudanças de inteligibilidade (15).

A variação de $1 \mathrm{~dB}$ na relação $S / R$ em pessoas com audição normal representa mudanças importantes de no reconhecimento da fala. São encontrados na literatura vários estudos que citam diferentes valores de variação para cada incremento favorável na relação sinal/ruído, como 18\% (9), 13,2\%(15) e 12,12\% (5), sendo este último pesquisado com o mesmo instrumento de avaliação utilizado no presente estudo.

Dessa forma, as diferenças entre os valores da relação $\mathrm{S} / \mathrm{R}$ entre os dois grupos aqui estudados são bastante relevantes, pois se usarmos o valor encontrado na pesquisa citada acima (5) que encontrou mudança no índice percentual de reconhecimento de fala $12,12 \%$ a cada variação de $1 \mathrm{~dB}$ na relação $\mathrm{S} / \mathrm{R}$, poderíamos projetar que os indivíduos do grupo $\mathrm{B}$, que necessitaram em torno de $3 \mathrm{~dB}$ de relação $\mathrm{S} / \mathrm{R}$ mais favorável para reconhecer $50 \%$ dos estímulos de fala, teriam índices percentuais de reconhecimento de fala no ruído em torno de $36,36 \%$ piores do que os indivíduos sem queixa (grupo A), se estivessem submetidos a uma mesma situação de comunicação, com uma relação S/R de -6 a $-7 \mathrm{~dB}$, por exemplo.

Outro dado constatado na literatura (16) é o valor de referência para a relação $S / R$ quando realizada a avaliação em fones auriculares, que foi de $-5,29 \mathrm{~dB}$ para adultos jovens normo-ouvintes, variando de $-2,55$ a $-9,22 \mathrm{~dB}, \mathrm{com}$ um desvio-padrão (DP) médio de $1,13 \mathrm{~dB}$. Considerando os dados da referida pesquisa e ponderando mais ou menos dois DP, a partir da média, chega-se a um valor mínimo encontrado para as relações $\mathrm{S} / \mathrm{R}$ de indivíduos jovens normo-ouvintes de- $3 \mathrm{~dB}$, tendo sido verificado que apenas um indivíduo necessitou de relação $\mathrm{S} / \mathrm{R}$ mais favorável do que $-3 \mathrm{~dB}$.

Por sua vez, na presente pesquisa, quando foram analisados os resultados individuais das relações $\mathrm{S} / \mathrm{R}$, constatou-se que todos os indivíduos do grupo A reconheceram $50 \%$ do material de fala apresentado com uma relação S/ $\mathrm{R}$ igual ou mais desfavorável do que $-3 \mathrm{~dB}$ em ambas as orelhas, sendo que os valores variaram entre -3,07 e-12,8 $\mathrm{dB}$ na OD e entre -3,66 e -11,77 dB na OE.

Já no grupo B, encontraram-se valores de -1,04 e $8,07 \mathrm{~dB}$ na OD e -0,75 e - 7,25 na OE. Apenas 17 (70\%) dos indivíduos alcançaram esse desempenho com relação $S / R$ mais desfavorável ou igual a - 3 dB na OD e 16 (66\%) na OE.

Isso mostra que os indivíduos que referem queixa para entender a fala em ambientes ruidosos realmente possuem maior dificuldade na tarefa de reconhecimento de sentenças no ruído, quando comparados a sujeitos que não relatam essa dificuldade com idade e características audiológicas semelhantes.

Compreender a fala em ambientes ruidosos é um desafio para qualquer ouvinte. Esta dificuldade é atribuída, em parte, aos efeitos negativos do ruído na sincronia neural, resultando em uma representação degradada da fala em níveis corticais e subcorticais (17).

Sujeitos com as mesmas habilidades de reconhecimento de fala no silêncio podem apresentar resultados extremamente diferentes em ambientes ruidosos. Quando a avaliação ocorre no ruído, ao contrário do silêncio, são exigidos vários canais auditivos para atingir o mesmo nível de reconhecimento da fala, indicando que informações 
sensoriais mais detalhadas são necessárias em condições de escuta difícil (2).

Esta tarefa requer um conjunto complexo de habilidades cognitivas e perceptuais, incluindo a memória de trabalho auditiva, detecção e processamento de aspectos espectrais e temporais (18, 19), além das habilidades auditivas de figura-fundo (20), fechamento auditivo e atenção seletiva (21).

Portanto, torna-se importante a avaliação da decodificação auditiva, pois qualquer prejuízo envolvido na aquisição de conhecimentos pela habilidade de integrar auditivamente a informação sonora dificultará a compreensão da fala em ambientes ruidosos (22).

Assim sendo, considerando todos estes aspectos e retomando os resultados da atual pesquisa - que evidenciou, através do teste LSP, que indivíduos audiologicamente normais, com queixa clínica de dificuldade para entender a fala no ruído, apresentaram desempenho inferior quando comparado a de indivíduos da mesma faixa etária, porém sem a queixa -, pode-se levantar a hipótese de que estes indivíduos possam apresentar prejuízo em alguma das etapas do processamento da fala e não conseguem realizar as habilidades de atenção seletiva figura-fundo com eficiência, levando ao baixo desempenho encontrado.

O objetivo da avaliação do reconhecimento de fala é a obtenção de uma compreensão abrangente sobre a forma de como a dificuldade auditiva afeta os diferentes processos envolvidos na compreensão da fala (23).

Se houver redução intrínseca combinada com a diminuição das pistas redundantes extrínsecas, a inteligibilidade será prejudicada. Os testes de reconhecimento de fala na presença de escuta difícil possibilitam avaliar as habilidades perceptuais auditivas e identificar uma alteração auditiva central (21).

Em vista do exposto, percebe-se que é de fundamental importância a avaliação do reconhecimento da fala da forma mais próxima possível das situações do dia-a-dia. Para isso, a utilização de ruído competitivo, que requer atividade auditiva complexa para que o estímulo de fala seja processado; e de testes que utilizam sentenças como estímulo, que simulam situações de comunicação na qual a extensão do enunciado a ser reconhecido e a complexidade linguística são fatores levados em consideração, mostram-se efetivos para estimar queixas clínicas relacionadas à dificuldade para entender a fala.

Então, para que se consiga mensurar a real dificuldade do indivíduo com queixa clínica de dificuldade para entender a fala no ruído, mesmo com limiares auditivos dentro da normalidade, sugere-se a introdução, na avaliação audiológica de rotina clínica, de testes que empregam sentenças na presença de ruído. Esta seria a forma mais confiável e eficiente de quantificar o desempenho das habilidades envolvidas neste processo. É uma abordagem mais abrangente, diferente da metodologia empregada nas avaliações atualmente realizadas, as quais avaliam o indivíduo somente em situações ideais de escuta, ou seja, no silêncio e com palavras isoladas, não demonstrando as reais dificuldades do paciente.

Deve ficar claro que dificuldades relacionadas à capacidade de extrair pistas acústicas da informação auditiva, focalizar a atenção na informação relevante e dificuldade de evocar a mensagem retida na memória de curto prazo influenciarão no desempenho dos indivíduos em ambientes que demandem essas habilidades. É importante ressaltar que este teste vai mostrar, confirmar e quantificar a dificuldade específica referida pelo paciente, norteando metas para uma possível intervenção e reabilitação.

A partir destes achados, quando o resultado for abaixo do esperado, o paciente será encaminhado para outras avaliações, tais como testes de processamento auditivo e exames eletrofisiológicos, quando possível, pois visam ratificar e complementar o diagnóstico para que, dessa forma, sejam fornecidas as orientações e sugestões de conduta terapêutica para auxiliar o paciente a minimizar a queixa clínica.

\section{ConClusão}

Com base nos resultados encontrados, pode-se concluir que indivíduos, normo-ouvintes, com queixa de dificuldade de entender a fala em ambientes ruidosos, apresentaram maior dificuldade na tarefa de reconhecimento de sentenças no ruído quando comparados a sujeitos que não relataram essa dificuldade, com idade e características audiológicas semelhantes.

Portanto, deve-se incluir na avaliação audiológica clínica de rotina testes que empregam sentenças na presença de ruído competitivo, pois esta é a forma mais confiável e eficiente de quantificar o desempenho dos indivíduos na tarefa de reconhecer a fala quando um ambiente sonoro é desfavorável.

A partir desta avaliação, acredita-se que é necessário investigar as habilidades de processamento auditivo quando houver queixa clínica de dificuldade para entender a fala no ruído mesmo quando o indivíduo apresenta audição normal, pois estes indivíduos podem apresentar algum déficit nas etapas do processamento da fala. 


\section{REFERÊNCIAS BIBLIOGRÁFICAS}

1. Theunissen M, Swanepoel DW, Hanekom J. Sentence recognition in noise: Variables in compilation and interpretation of tests. Int J Audiol. 2009, 48:743-757.

2. Ziegler JC, Pech-George C, George F,Lorenzi C. Speech perception in noise déficits in dyslexia. Dev Sci. 2009, 12:73245 .

3. Paula A, Oliveira JAP, Godoy NM. Baixa discriminação auditiva em ambiente competitivo de pacientes jovens com audiograma normal. Rev Bras de Otorrinolaringol. 2000, 66(5):439-42.

4. Miranda EC, Costa MJ. Reconhecimento de sentenças no silêncio e no ruído em indivíduos jovens adultos normo-ouvintes em campo livre. Fono Atual. 2006, 8(35):4-12.

5. Henriques MO, Costa MJ. Limiares de reconhecimento de sentenças no ruído, em campo livre: valores de referência para adultos normo-ouvintes. Rev Bras de Otorrinolaringol. 2008, 74(2):188-92.

6. Freitas CD, Lopes LFD, Costa MJ. Confabilidade dos limiares de reconhecimento de sentenças no silêncio e no ruído. Rev Bras Otorrinolaringol. 2005, 71(5):624-32.

7. Costa, MJ. Listas de sentenças em português: apresentação e estratégias de aplicação na audiologia. Santa Maria: Pallotti; 1998.

8. Davis H, Silverman SR. Interpretação dos resultados da avaliação audiológica. In: Santos TMM, Russo ICP. Prática da audiologia clínica. 6ª ed. São Paulo: Cortez; 2007. p. 291310.
9. Smoorenburg GF. Speech reception in quiet and in noise conditions by individuals with noise-induced hearing loss in relation to their tone audiogram. J Acoust Soc Am. 1992, 91(1):421-37.

10. Levitt H, Rabiner LR. Use of a sequencial strategy in intelligibility testing. J Acoust Soc Am. 1967, 42:609-12.

11. Cóser PL, Costa MJ, Cóser MJS, Fukuda Y. Reconhecimento de sentenças no silêncio e no ruído em indivíduos portadores de perda induzida pelo ruído. Rev Bras de Otorrinolaringol. 2000, 66(4):362-70.

12. Middelweerd MJ, Festen JM, Plomp R. Difficulties with speech intelligibility in noise in spite of a normal pure-tone audiogram. Int J Audiol. 1990, 29(1):1-7.

13. Soncini F, Costa MJ, Oliveira TT. Queixa de dificuldade para reconhecer a fala $\mathrm{X}$ limiares de reconhecimento de fala no ruído em normo-ouvintes com mais de 50 anos. Fono Atual. 2003, 26:4-11.

14 Caporali SA, Arieta AM. Reconhecimento de fala: estudo comparativo entre grupos com e sem queixa de percepção da fala. Rev Soc Bras Fonoaudiol. 2004, 9(3):129-35.

15 Wagener KC. Factors influencing sentence intelligibility in noise. Bibliotheks- und Information System der Universität Oldenburg, 2004.

16 Costa MJ, Daniel RC, Santos SN. Reconhecimento de sentenças no silêncio e no ruído em fones auriculares: valores de referência de normalidade. Rev. CEFAC; São Paulo, 2010. doi: 10.1590/S1516-18462010005000114.

17 Anderson S, Skoe E, Chandrasekaran B, Kraus N. Neural Timing is Linked to Speech Perception in Noise. The Journal of Neuroscience. 2010, 30(14):4922-26. 